Electromagnetic-induced transparency of Wannier-Mott excitons

This content has been downloaded from IOPscience. Please scroll down to see the full text.

View the table of contents for this issue, or go to the journal homepage for more

Download details:

IP Address: 192.167.18.242

This content was downloaded on 07/02/2015 at 11:53

Please note that terms and conditions apply. 


\title{
Electromagnetic-induced transparency of Wannier-Mott excitons
}

\author{
M. Artoni ${ }^{1}$, G. C. LA RoccA ${ }^{2,3}$ and F. Bassani ${ }^{2}$ \\ 1 INFM, European Laboratory for Non-linear Spectroscopy \\ Largo E. Fermi 2, Florence, Italy \\ 2 INFM, Scuola Normale Superiore - Piazza dei Cavalieri 7, 56126 Pisa, Italy \\ 3 Dipartimento di Fisica, Università di Salerno - 84081 Baronissi (Sa), Italy
}

(received 12 August 1999; accepted in final form 5 December 1999)

PACS. 42.50.-p - Quantum optics.

\begin{abstract}
We predict a remarkable quenching of the absorption due to electromagneticinduced transparency in an undoped bulk semiconductor. For free-exciton lines the effect is expected to be as large as that observed in atomic systems. The conditions for its occurrence are determined and numerical estimates are presented for the specific case of the "yellow" exciton of $\mathrm{Cu}_{2} \mathrm{O}$.
\end{abstract}

Electromagnetic-induced transparency is one of today's most thoroughly studied examples of coherence and quantum interference: it has been so far demonstrated mostly for dilute atomic vapors [1]. The lower doublet of a three-level system may be pumped into a coherent superposition state (population trapping state) where the population is trapped due to destructive interference between two distinct paths of absorption to the third level [2]. Condensed matter exhibits quite a variety of three-level systems where induced transparency could be achieved in much the same way as done with atoms. Yet dephasings, which can easily break the coherence of the population trapping state, are typically much faster in solids than in atomic vapors; this has made it difficult to observe a large electromagnetic-induced transparency effect in solids. Very few cases have been so far reported. Microwave-induced transparency has been observed in ruby [3] where the energy separation of the required threelevel configuration was obtained by using a strong external magnetic field. Optical-induced transparency has instead been observed in an inhomogeneously broadened hole-burning $\mathrm{Pr}^{3+}$ doped $\mathrm{Y}_{2} \mathrm{SiO}_{5}$ crystal through a direct measurement of the suppression of absorption [4] and also through a measurement of quenching of the excited-state fluorescence [5].

In this work, we predict a remarkable enhancement of electromagnetic-induced transparency in an undoped bulk semiconductor exhibiting sharp free-exciton lines that correspond to intrinsic delocalized electronic states. We specifically consider the "yellow exciton" series in $\mathrm{Cu}_{2} \mathrm{O}$ for which a $\Lambda$-type model Hamiltonian will be developed. The reason for such a choice is two-fold, namely, all the spectroscopic parameters are well known [6] and the relevant pump and probe set-up required for the demonstration of electromagnetic-induced transparency has already been employed to investigate the optical Stark effect in $\mathrm{Cu}_{2} \mathrm{O}$ [7].

(c) EDP Sciences 


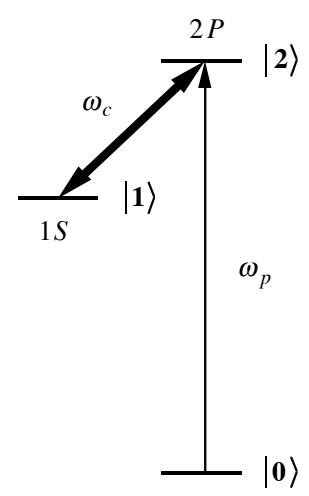

Fig. 1 - Schematic diagram of the exciton states for the yellow series in $\mathrm{Cu}_{2} \mathrm{O}$.

The optical properties of a semiconductor are mainly determined by the lowest conduction and uppermost valence bands. We take non-degenerate parabolic bands in a direct-gap intrinsic semiconductor with band extrema at $\vec{k}=0$ ( $\Gamma$ point), as appropriate for $\mathrm{Cu}_{2} \mathrm{O}[8,9]$. A weak probe beam with an electric field $\vec{E}_{\mathrm{p}}$ of frequency $\omega_{\mathrm{p}}$ close to the band-gap frequency transition $E_{\mathrm{g}} / \hbar$ and a strong coupling beam with a field $\vec{E}_{\mathrm{c}}$ of frequency $\omega_{\mathrm{c}}$ much smaller than $E_{\mathrm{g}} / \hbar$ couple, respectively, to inter-band and intra-band transitions. Both fields are assumed to be coherent and monochromatic plane waves whose wave vectors are negligible compared to the electronic ones and only vertical transitions will be examined in the following. We adopt the two-band approximation for both inter- and intra-band transitions [8]. Although our analysis will be carried out for continuous waves, for the properties derived here the same results hold true for a pump pulse long enough to maintain the required coherence during the passage through the sample of a synchronized shorter probe pulse. Yet, the latter should be made of sufficient duration that its Fourier components are contained within the sample transparency window. In the velocity gauge $(\vec{A} \cdot \vec{p})$, the relevant semiclassical interaction Hamiltonian $H_{\mathrm{I}}=H_{\mathrm{I}}^{\mathrm{p}}+H_{\mathrm{I}}^{\mathrm{c}}$ can be written as (the $A^{2}$ term is negligible)

$$
H_{\mathrm{I}}^{\mathrm{p}}=-\frac{i e E_{p} \hat{\epsilon}_{p} e^{-i \omega_{\mathrm{p}} t}+\text { c.c. }}{m_{0} \omega_{\mathrm{p}}} \sum_{k, q}\left(\langle c, q|\vec{p}| v, k\rangle c_{q}^{\dagger} h_{-k}^{\dagger}-\langle v, q|\vec{p}| c, k\rangle c_{k} h_{-q}\right)
$$

and

$$
H_{\mathrm{I}}^{\mathrm{c}}=-\frac{i e E_{\mathrm{c}} \hat{\epsilon}_{\mathrm{c}} e^{-i \omega_{\mathrm{c}} t}+\text { c.c. }}{m_{0} \omega_{\mathrm{c}}} \sum_{k, q}\left(\langle c, q|\vec{p}| c, k\rangle c_{q}^{\dagger} c_{k}-\langle v, q|\vec{p}| v, k\rangle h_{-k}^{\dagger} h_{-q}\right)
$$

where $m_{0}$ is the electron rest mass. We denote by $c$ and $c^{\dagger}\left(h\right.$ and $\left.h^{\dagger}\right)$ the destruction and creation operators for electrons (holes) while the matrix elements of the momentum operator $\vec{p}$ are taken between Bloch states in the conduction (valence) band.

Since the probe is weak and the coupling beam cannot induce inter-band transitions, no significant population can be created in the excited levels; in this case phase-space filling, bandgap renormalization and exciton blue-shift do not occur [10] and the Coulomb interaction gives rise to well-defined exciton levels that dominate the optical response. In the case of $\mathrm{Cu}_{2} \mathrm{O}\left(\mathrm{O}_{h}\right.$ symmetry) such levels exhibit a hydrogenic yellow exciton series associated with the lowest inter-band transition between the $\Gamma_{7}^{+}$valence band and the $\Gamma_{6}^{+}$conduction band. Because both band-edge states are of even parity, the lowest $1 S$ exciton state is dipole-forbidden, whereas the $2 P$ state is dipole-allowed; the $1 S$ to $2 P$ transition is also allowed. The Hamiltonian 
describing this three-level exciton configuration (see fig. 1) can be determined from the usual envelope function picture of Wannier-Mott exciton states [8-10] according to which $|1\rangle \equiv$ $|1 S, Q \simeq 0\rangle=\sum_{k} \phi_{1 S}(\vec{k}) c_{k}^{\dagger} h_{-k}^{\dagger}|0\rangle$ and a similar expression holds for the $2 P$ exciton state $|2\rangle$. As usual $|0\rangle$ is the vacuum state with an empty conduction band and a filled valence band while the $\phi(\vec{k})$ 's are the Fourier transforms of the envelope functions for the relative motion. The center-of-mass wave vectors $\vec{Q}$ have been taken to vanish since only excitons with a negligible total wave vector couple to light. In the rotating-wave approximation, one has

$$
\left\langle 2\left|H_{\mathrm{I}}\right| 0\right\rangle=\frac{e E_{\mathrm{p}} e^{-i \omega_{\mathrm{p}} t}}{i m_{0} \omega_{\mathrm{p}}} \sum_{k}\left\langle c, k\left|\hat{\epsilon}_{\mathrm{p}} \cdot \vec{p}\right| v, k\right\rangle \phi_{2 P}^{*}(\vec{k})
$$

and

$$
\left\langle 2\left|H_{\mathrm{I}}\right| 1\right\rangle=\frac{e E_{\mathrm{c}} e^{-i \omega_{\mathrm{c}} t}}{i m_{0} \omega_{\mathrm{c}}} \sum_{k} \hat{\epsilon}_{\mathrm{c}} \cdot(\langle c, k|\vec{p}| c, k\rangle-\langle v, k|\vec{p}| v, k\rangle) \phi_{2 P}^{*}(\vec{k}) \phi_{1 S}(\vec{k}) .
$$

The matrix elements above for a non-vanishing wave vector $\vec{k}$ near the $\Gamma$ point can be evaluated using the standard $\vec{k} \cdot \vec{p}$ perturbation theory $[8,9]$. For a probe beam polarized along $\hat{x}$ eq. (3) becomes

$$
\begin{aligned}
\left\langle 2\left|H_{\mathrm{I}}\right| 0\right\rangle & =\frac{\hbar \beta e E_{\mathrm{p}} e^{-i \omega_{\mathrm{p}} t}}{i m_{0} \omega_{\mathrm{p}}} \sum_{k}\left(k_{y}+k_{z}\right) \phi_{2 P}^{*}(\vec{k})= \\
& =\left.\frac{\hbar \beta e E_{\mathrm{p}} e^{-i \omega_{\mathrm{p}} t}}{m_{0} \omega_{\mathrm{p}}} \sqrt{V}\left(\partial_{y}+\partial_{z}\right) \phi_{2 P}^{*}(\vec{r})\right|_{r=0} \equiv-E_{\mathrm{p}} e^{-i \omega_{\mathrm{p}} t}\left(D_{20 y}+D_{20 z}\right),
\end{aligned}
$$

where $V$ is the crystal volume and $\phi(\vec{r}=0)$ is of the order of $a_{\mathrm{B}}^{-3 / 2}$ ( $a_{\mathrm{B}}$ being the exciton Bohr radius). The dimensionless coefficient $\beta$ is formally given by a summation over all bands except the $\Gamma_{6}^{+}$conduction and $\Gamma_{7}^{+}$valence bands and will be later estimated from the measured value of the oscillator strength. From the symmetry of the band-edge states, it follows that a probe beam polarized along $\hat{x}$ couples equally well to the $2 P_{y}$ and $2 P_{z}$ excitons, but not to the $2 P_{x}$ one. As for (3) we can rewrite (4) as

$$
\begin{aligned}
\left\langle 2\left|H_{I}\right| 1\right\rangle & =\frac{\hbar e E_{\mathrm{c}} e^{-i \omega_{\mathrm{c}} t}}{i \mu \omega_{\mathrm{c}}} \sum_{k} \vec{k} \cdot \hat{\epsilon}_{\mathrm{c}} \phi_{2 P}^{*}(\vec{k}) \phi_{1 S}(\vec{k})= \\
& =e E_{\mathrm{c}} e^{-i \omega_{\mathrm{c}} t} \hat{\epsilon}_{\mathrm{c}} \cdot \int \mathrm{d}^{3} r \phi_{2 P}^{*}(\vec{r}) \vec{r} \phi_{1 S}(\vec{r}) \equiv-E_{\mathrm{c}} e^{-i \omega_{\mathrm{c}} t} \hat{\epsilon}_{\mathrm{c}} \cdot \vec{D}_{21},
\end{aligned}
$$

where use has been made of the relation $i \hbar \vec{p} / \mu=\left[\vec{r}, H_{\text {eff }}\right]$. Here $H_{\text {eff }}$ the effective mass Hamiltonian for the exciton relative motion with eigenfunctions $\phi_{1 S}$ and $\phi_{2 P}$ and $\mu=m_{\mathrm{e}} m_{\mathrm{h}} /\left(m_{\mathrm{e}}+\right.$ $m_{\mathrm{h}}$ ) is the reduced mass in terms of the electron and hole effective mass. The expression for $\vec{D}_{21}$ is analogous to that for an atomic transition and an estimate by using hydrogenic wave functions with appropriate Bohr radii yields $\left|\vec{D}_{21}\right| \simeq|e| a_{\mathrm{B}} \simeq 30$ Debye. If the probe beam is polarized along $\hat{x}$, only a coupling beam polarized along $\hat{y}$ or $\hat{z}$ will couple to the same $2 P$ exciton levels.

In the following we will restrict ourselves to a probe and a coupling beam polarized, respectively, along $\hat{x}$ and $\hat{y}$. The total Hamiltonian, after a transformation to a rotating frame so as to eliminate the coupling and probe free field terms, can be cast in the form

$$
\begin{aligned}
H= & \hbar \omega_{1 S}|1\rangle\left\langle 1\left|+\hbar \omega_{2 P}\right| 2\right\rangle\langle 2|- \\
& -\left(E_{\mathrm{p}} e^{-i \omega_{\mathrm{p}} t} D_{20 y}|2\rangle\left\langle 0\left|+E_{\mathrm{c}} e^{-i \omega_{\mathrm{c}} t} D_{21 y}\right| 2\right\rangle\langle 1|+\text { h.c. }\right),
\end{aligned}
$$


where the state $|1\rangle$ and $|2\rangle$ refer now to $1 S$ and $2 P_{y}$ excitons. The derivation of the $D$ 's outlined above takes into account the microscopic details of the relevant excitonic states but neglect many-body effects as they were not included in the original fermionic equations (1) and (2).

Characteristic absorptive properties are assessed through the study of the imaginary part of the relevant (non-linear) susceptibility. This is in turn related to the off-diagonal elements of the reduced density matrix $\rho$ for the electronic system the evolution of which is governed by the master equation [11],

$$
\dot{\rho}=-\frac{i}{\hbar}[H, \rho]-\frac{1}{2}\{T, \rho\} .
$$

The second term on the right-hand side, containing the diagonal relaxation operator $T$, describes the damping of the exciton states through phenomenological relaxation rates $T_{i i}$ $(i=0,1,2)$ with $T_{00}=0$. These rates are determined by exciton-damping mechanisms comprising, besides the very small radiative damping, temperature-dependent homogeneous broadening due to phonons and inhomogeneous broadening due to structural imperfections and impurities. In our case, $\rho_{11}$ and $\rho_{22}$ are negligible, excitons behave as bosons, and a straightforward calculation leads to an analytical steady-state solution for the off-diagonal elements of $\rho$. By taking the leading order in the probe field $E_{\mathrm{p}}$, but keeping the coupling field $E_{\mathrm{c}}$ to all orders, we can write the steady-state complex exciton susceptibility exhibited to the incident probe in the following form:

$$
\begin{aligned}
\chi_{\mathrm{p}}\left(\omega_{\mathrm{p}}\right) & =\frac{\tilde{\rho}_{02} D_{20 y}^{*}}{\epsilon_{0} V E_{\mathrm{p}}} \\
& =A \frac{\gamma_{2 P}}{\left(\delta_{\mathrm{p}}-i \gamma_{2 P}\right)}\left[1-\frac{\Omega_{\mathrm{c}}^{2} / 4}{\left(\delta_{\mathrm{p}}-i \gamma_{2 P}\right)\left(\delta_{\mathrm{p}}-\delta_{\mathrm{c}}-i \gamma_{1 S}\right)}\right]^{-1},
\end{aligned}
$$

where $\tilde{\rho}_{02}=\rho_{02} e^{i \omega_{\mathrm{p}} t}$ and $\gamma_{2 P} \simeq T_{22} / 2$ and $\gamma_{1 S} \simeq T_{11} / 2$ are the overall dephasing rates of the two exciton states. We denote here by $\delta_{\mathrm{p}}=\omega_{2 P}-\omega_{\mathrm{p}}$ and $\delta_{\mathrm{c}}=\omega_{2 P}-\omega_{1 S}-\omega_{c} \equiv \delta_{2 P-1 S}-\omega_{c}$, respectively, the probe and coupling beam detunings; $\Omega_{c}=2\left|E_{\mathrm{c}} D_{21 y}\right| / \hbar$ is the coupling beam effective Rabi frequency. The splitting $\delta_{2 P-1 S}$ and the coefficient $A=\left|D_{20 y}\right|^{2} /\left(\epsilon_{0} \hbar \gamma_{2 P} V\right)$ depends on the material parameters, $\left|D_{20 y}\right|^{2}$ being proportional to the $2 P$ exciton oscillator strength. For the yellow exciton series of bulk $\mathrm{Cu}_{2} \mathrm{O}$ one has $\hbar \omega_{2 P} \simeq 2.148 \mathrm{eV}$ and $\hbar \delta_{2 P-1 S}=$ $115 \mathrm{meV}$ [7], while from the measured linewidth $\hbar \gamma_{2 P} \simeq 1 \mathrm{meV}$ [7] and oscillator strength [6] the coefficient $A$ can be estimated to be $A \simeq 0.02$. Such a small value, due to the fact that the $2 P$ exciton line is rather weak (second-class transition) [8], is comparable to that of cold atomic gases where $A$ commonly varies in the range $10^{-2}-10^{-1}$.

We now use the imaginary part of $\chi_{\mathrm{p}}$ to establish changes in the $\mathrm{Cu}_{2} \mathrm{O}$ absorption due to electromagnetic-induced transparency. We show in fig. 2 both cases of a resonant and a non-resonant pump: in the absence of the coupling beam the probe is absorbed (grey curve) while in its presence increasing degrees of transparency are observed to depend on increasing values of the coupling beam intensity for a fixed detuning. The coupling laser that couples the levels $|1\rangle$ and $|2\rangle$ splits the probe absorption line into two components each centered at the transition frequencies from the common ground $|0\rangle$ to two dressed excited states created by the coupling beam [12]. These two components, whose widths are essentially unmodified for reasonably modest pumps intensities (negligible power broadening), are instead separated by an amount that is equal to the (ac) Stark splitting $\Omega_{\mathrm{R}}=\sqrt{\Omega_{\mathrm{c}}^{2}+\delta_{\mathrm{c}}^{2}}$. For small Stark splittings, roughly not exceeding twice the value of the dephasing $\gamma_{2 P}$ of the $2 P$ exciton, we 

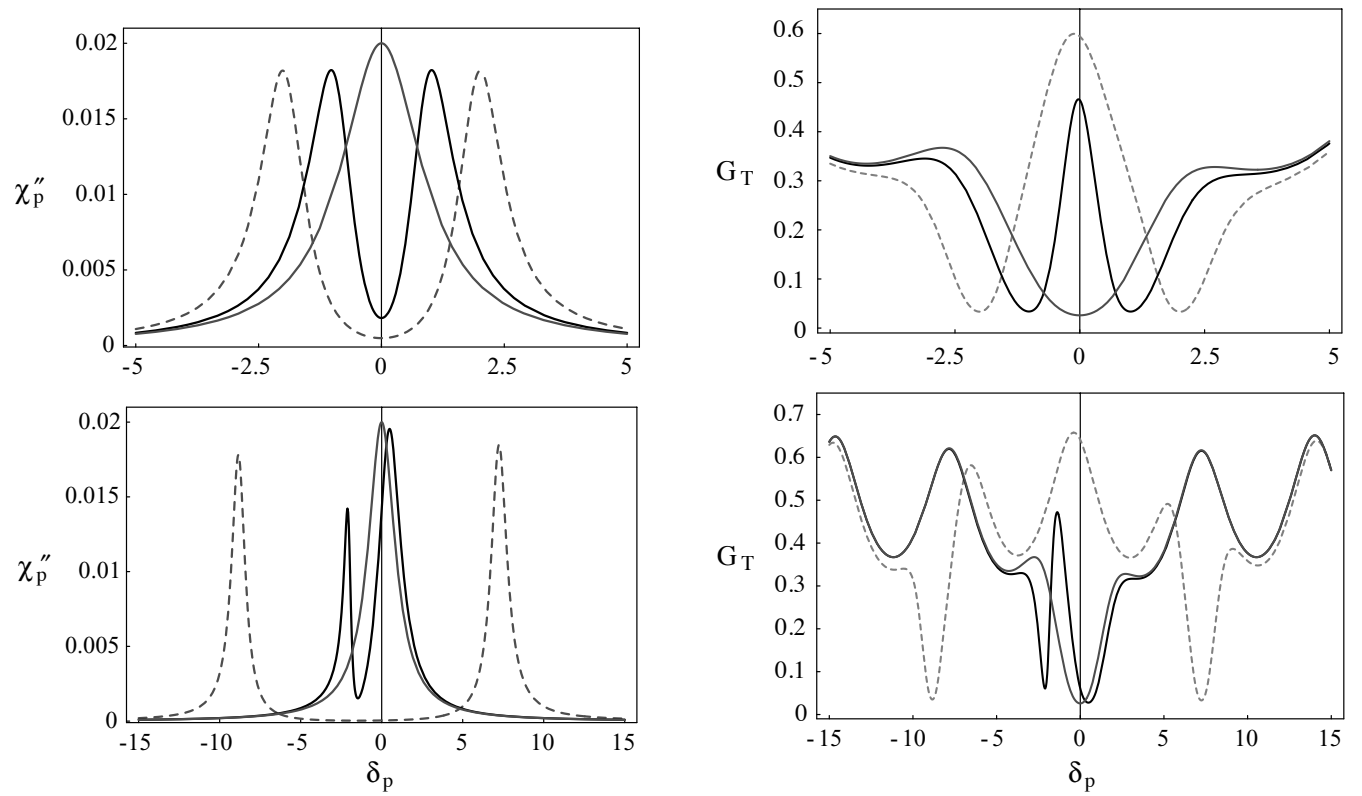

Fig. 2

Fig. 3

Fig. 2 - Imaginary part of the susceptibility vs. the probe frequency detuning in units of $\gamma_{2 P}$ $\left(\hbar \gamma_{2 P}=1 \mathrm{meV}\right)$. The upper and lower frame refer, respectively, to a resonant and a non-resonant coupling beam with detuning $\delta_{\mathrm{c}} / \gamma_{2 P}=-1$. The coupling Rabi frequencies are (upper) $\Omega_{\mathrm{c}} / \gamma_{2 P}=2$ (black solid), 4 (grey dash) and (lower) $\Omega_{\mathrm{c}} / \gamma_{2 P}=2$ (black solid), 16 (grey dash) which correspond, respectively, to electric field amplitudes (upper) $E_{\mathrm{c}}=16 \mathrm{KV} / \mathrm{cm}$ (black solid), $32 \mathrm{KV} / \mathrm{cm}$ (grey dash) and (lower) $E_{\mathrm{c}}=16 \mathrm{KV} / \mathrm{cm}$ (black solid), $256 \mathrm{KV} / \mathrm{cm}$ (grey dash). The $1 S$ exciton dephasing is $\gamma_{1 S} / \gamma_{2 P}=0.1$ and $A=2 \times 10^{-2}$. In the absence of the coupling beam the susceptibility is described by the solid grey curve.

Fig. 3 - Transmission coefficient $G_{\mathrm{T}}$ vs. the probe frequency detuning in units of $\gamma_{2 P}$ for a resonant (upper frame) and a non-resonant coupling beam (lower frame) with detuning $\delta_{\mathrm{c}} / \gamma_{2 P}=-1.6$. The bulk dielectric constant is $\epsilon_{\infty}=6.5+i 2 \times 10^{-3}$, the slab thickness $d=35 \mu \mathrm{m}$, while the other parameters are the same as in the corresponding frames of fig. 2 . In the upper frame we only report the transmission on line-center.

observe a minimum in the absorption whenever the probe detuning equals that of the pump, i.e., $\omega_{\mathrm{p}}-\omega_{\mathrm{c}}=\omega_{1 S}$. The fact that the minimum occurs at the equal-detuning frequency is a manifestation of the optically induced coherence over the non-allowed transition between the ground and the $1 S$ level. Under these conditions such a reduction can be explained in terms of electromagnetic-induced transparency, i.e., as a combination of the Stark splitting and the destructive quantum interference between two different absorption paths from the common ground $|0\rangle$ to the two dressed excited states. One could expect that (incoherent) bleaching or saturation effects would yield a similar absorption minimum; yet, these effects would be maximized for a probe close to resonance and not when it has the same detuning of the coupling beam. For large Stark splittings, on the other hand, the absorption is very small between the two Autler-Townes sidebands. Such a uniform quenching does not originate from quantum interference because the upper doublet of dressed levels is now too far apart for the interference to take place. 
The direct observation of induced transparency hinges on the actual transmission efficiency. We then proceed to evaluate the probe transmission intensity coefficient $G_{\mathrm{T}}$ specific for a $\mathrm{Cu}_{2} \mathrm{O}$ sample. For normal incidence across a slab of thickness $d$, one has

$$
G_{\mathrm{T}}(\omega)=\frac{16\left|n_{\mathrm{p}}(\omega)\right|^{2} \exp \left[-2 \omega \kappa_{\mathrm{p}}(\omega) d / c\right]}{\left|\left(n_{\mathrm{p}}(\omega)+1\right)^{2}-\left(n_{\mathrm{p}}(\omega)-1\right)^{2} \exp \left[2 i \omega n_{\mathrm{p}}(\omega) d / c\right]\right|^{2}},
$$

where $n_{\mathrm{p}}(\omega)=\sqrt{\epsilon_{\infty}+\chi_{\mathrm{p}}(\omega)}=\eta_{\mathrm{p}}(\omega)+i \kappa_{\mathrm{p}}(\omega)$ is the complex refractive index, and $\epsilon_{\infty}$ is the complex background dielectric constant. The imaginary part $\epsilon_{\infty}^{\prime \prime}$ accounts for the background absorption to which the exciton absorption line is always superimposed. It originates mainly from indirect transitions, surface and impurity scattering and is directly related to the background value $\kappa_{\infty}$ of the extinction coefficient. The latter can be estimated from the logarithm of the measured exciton peak transmission relative to the background value; from [6] we can infer an average value $\kappa_{\infty} \simeq 4 \times 10^{-4}$ over the $2 P$ exciton absorption linewidth yielding $\epsilon_{\infty}^{\prime \prime}=2 \times 10^{-3}$.

With the help of (9) we evaluate $G_{\mathrm{T}}$ which we show in fig. 3 for typical experimental parameters. The quantum interference modifies the transmission linewidth in a non-trivial way; since $\gamma_{1 S} \simeq 0.1 \mathrm{meV}[7]$ can only in part be controlled by reducing the temperature and by increasing the sample structural quality, the detunings and the coupling Rabi frequency remain the essential key parameters to tailor the interference. In the absence of the pump, transmission would be quite small $\left(G_{\mathrm{T}} \sim 10^{-2}\right)$, whereas when the coupling beam is on we can distinguish between two transparency regimes: one due to a large Stark splitting and the other due to quantum interference taking place respectively for strong and weak coupling intensities.

For weak pumps, regardless of their detunings, the height of the transparency window increases appreciably more than its width does, making the absorption drop nearly $50 \%$. The transmission linewidths are always subnatural $\left(<\gamma_{2 P}\right)$, which is a typical feature of the electromagnetic-induced transparency. Higher degrees of transparency are restrained more by the broadening of the $1 S$ to ground transition than by smaller thicknesses or higher coupling laser intensities; smaller d's would simply make the sample transparent altogether, while high peak-power densities would make the interference hard to achieve. On the other hand, by reducing $\gamma_{1 S}$ to half its value would produce a further $20 \%$ increase in the window height $\left(G_{\mathrm{T}} \simeq 0.55\right)$, while its width would narrow down to nearly a third of $\gamma_{2 P}$. Conversely, for sufficiently intense pumps, the transparency width increases more than the height does, marking the transition to a large ac Stark splitting comprising a wide transparency band separated by two absorption dips in correspondence of the Autler-Townes sidebands. Notice that the transmission band exhibits in this case the typical oscillating structure due to multiple internal reflections [13].

In this letter, we propose an experiment to observe fully developed electromagnetic-induced transparency in an undoped semiconductor, based on the well-known Wannier-Mott exciton lines. Unlike atomic vapours, electromagnetic-induced transparency in semiconductors would offer the exciting opportunity for device applications. The situation might be particularly favorable for low-dimensional semiconductor structures [14] and microcavities where the effect of induced transparency could easily be tailored.

We would like to thank F. Bogani for helpul and stimulating discussions. MA thanks the INFM for financial support. The work was supported in part by UNESCO (grant UVOROSTE 875.563.9). 


\section{REFERENCES}

[1] Harris S., Phys. Today, 50 (1997) 36; Arimondo E., Progress in Optics, edited by E. Wolf (Elsevier Science) 1996, p. 257; and references therein.

[2] Alzetta G., Gozzini A., Moi L. and Orriols G., Nuovo Cimento B, 36 (1976) 5; Arimondo E. and Orriols G., Lett. Nuovo Cimento, 17 (1976) 333.

[3] Zhao Y., Wu C., Ham B. S., Kim M. K. and Awad E., Phys. Rev. Lett., 79 (1997) 641.

[4] Ham B. S., Shahriar M. S. and Hemmer R. P., Opt. Lett., 22 (1997) 1138.

[5] Ichimura K., Yamamoto K. and Gemma N., Phys Rev. A, 58 (1998) 4116.

[6] Nikitine S., Grun J. B., Sieskind M., J. Phys. Chem. Solids, 17 (1961) 292; LandoltBÖRnsteIn, series III, 17 (Semiconductors) (Springer, Berlin), 1984.

[7] Fröhlich D., Nothe A. and Reimann K., Phys. Rev. Lett., 55 (1985) 1335; Fröhlich D., Neumann Ch., Uebbing B. and Wille R., Phys. Status Solidi B, 159 (1990) 297.

[8] Bassani F. and Pastori G. Parravicini, in Electronic States and Optical Transitions in Solids (Pergamon Press, Oxford) 1975; P. YU and M. CARdonA, Fundamentals of Semiconductors (Springer, Berlin) 1996.

[9] Elliot R. J., Phys Rev., 108 (1957) 1384; 124 (1959) 304.

[10] Haug H. and Koch S., Quantum Theory of the Optical and Electronic Properties of Semiconductors (World Scientific, Singapore) 1990.

[11] Scully M. O. and Zubairy S., Quantum Optics (Cambrigde University Press) 1998.

[12] Cohen-Tannoudji C., Dupont-Roc J. and Grynberg G., Atom-Photon Interactions (J. Wiley and Sons, Inc.) 1992.

[13] Born M. and Wolf E., Principles of Optics, sixth edition (Pergamon Press) 1993.

[14] See, e.g., Sadeghi S. M., Young J. F. and Meyer J., Phys. Rev. B., 56 (1997) 15557. 